\title{
Daily variations in air pollution and respiratory health in a multicentre study: the PEACE project
}

\author{
W. Roemer*, G. Hoek*, B. Brunekreef*+, J. Haluszka++, A. Kalandidi\#, J. Pekkanen \\ for the PEACE project
}

\begin{abstract}
Daily variations in air pollution and respiratory health in a multicentre study: the PEACE project. W. Roemer, G. Hoek, B. Brunekreef, J. Haluszka, A. Kalandidi, J. Pekkanen, for the PEACE project. COERS Journals Ltd 1998.

ABSTRACT: The Pollution Effects on Asthmatic Children in Europe (PEACE) study is a multicentre study of the acute effects of particles with a $50 \%$ cut-off aerodynamic diameter of $10 \mu \mathrm{m}(\mathrm{PM} 10)$, black smoke (BS), sulphur dioxide $\left(\mathrm{SO}_{2}\right)$ and nitrogen dioxide $\left(\mathrm{NO}_{2}\right)$ on the respiratory health of children with chronic respiratory symptoms.
\end{abstract}

The study was conducted in the winter of $1993 / 1994$ by 14 research centres in Europe. A total of 2,010 children, divided over 28 panels in urban and suburban locations, was followed for at least 2 months. Exposure to air pollution was monitored on a daily basis. Health status was monitored by daily peak expiratory flow (PEF) measurements and a symptom diary. The association between respiratory health and air pollution levels was calculated with time series analysis. Combined effect estimates of air pollution on PEF or the daily prevalence of respiratory symptoms and bronchodilator use were calculated from the panel-specific effect estimates. Fixed effect models were used and, in cases of heterogeneity, random effect models.

No clear associations between PM10, BS, $\mathrm{SO}_{2}$ or $\mathrm{NO}_{2}$ and morning PEF, evening PEF, prevalence of respiratory symptoms or bronchodilator use could be detected. Only previous day PM10 was negatively associated with evening PEF, but only in locations where BS was high compared to PM10 concentrations. There were no consistent differences in effect estimates between subgroups based on urban versus suburban, geographical location or mean levels of $\mathrm{PM10}, \mathrm{BS}, \mathrm{SO}_{2}$ and $\mathrm{NO}_{2}$. The lack of association could not be attributed to a lack of statistical power, low levels of exposure or incorrect trend specifications.

In conclusion, the PEACE project did not show effects of particles with a 50\% cutoff aerodynamic diameter of $\mathbf{1 0} \mu \mathrm{m}$, black smoke, sulphur dioxide or nitrogen dioxide on morning or evening peak expiratory flow or the daily prevalence of respiratory symptoms and bronchodilator use.

Eur Respir J 1998; 12: 1354-1361.
*Environmental and Occupational Health Group, Agricultural University Wageningen, The Netherlands. +Coordinator, PEACE project. ${ }^{++}$Tbc and Lung Diseases National Institute, Paediatric Faculty, Rabka, Poland. "Dept of Hygiene and Epidemiology, Medical School, University of Athens, Greece. ${ }^{*}$ National Public Health Institute, Kuopio, Finland

Correspondence: W. Roemer, Environmental and Occupational Health Group, Agricultural University Wageningen, P.O. Box 238, $6700 \mathrm{AE}$ Wageningen, The Netherlands. Fax: 31317485278

Keywords: Air pollution, children, epidemiology, particulate matter, peak expiratory flow variability, respiratory symptoms

Received: January 301998

Accepted after revision June 301998

The PEACE study was funded in the framework of the Commission of the European Communities Environment Programme, contracts EV5V-CT92-0220, CIPD-CT-92-5052 and ERBCIPD-CT93-0046. The Finnish, Norwegian and two Swedish centres were funded by grants from the respective governments. The study was co-ordinated by the Environmental and Occupational Health Group, Agricultural University Wageningen, The Netherlands.
Recent studies have demonstrated acute effects of air pollution on pulmonary function [1-5], respiratory symptoms $[1-3,5]$ and medication use $[1,3]$ in groups of children, using air pollution indicators such as particles with a $50 \%$ cut-off aerodynamic diameter of $10 \mu \mathrm{m}$ (PM10), sulphur dioxide $\left(\mathrm{SO}_{2}\right)$ and black smoke (BS). In some of these studies $[4,5]$ it was not possible to disentangle the effects of the separate air pollution indicators. Effects could be seen below the 1987 World Health Organization (WHO) air-quality guidelines [6]. These WHO air quality guidelines for Europe were based on older epidemiological studies using indicator pollutants such as total suspended particulates (TSP), $\mathrm{BS}$ and $\mathrm{SO}_{2}$, at higher concentrations of these pollutants than encountered in most European areas today. The concentration and composition of air pollution have changed over the last decades in many areas of Europe [7]. Components such as $\mathrm{SO}_{2}$ and airborne, coarse particulates have decreased owing to emission abatement measures and changes in energy production, industrial processes and space heating. Levels of other pollutants such as nitrogen dioxide $\left(\mathrm{NO}_{2}\right)$ and ozone $\left(\mathrm{O}_{3}\right)$ have increased during the same period, mostly through increased motor vehicle traffic. Thus, information is needed to evaluate how the response to air pollution depends on the composition of the current air pollution mixture. The possible difference in response to air pollution characterized by high

Other panel members: J. Heinrich (GSF-Institute of Epidemiology, Neuherberg, Germany), F. Kotesovec (District Institute of Hygiene, Teplice, Czech Republic), G. Niepsuj (Silesian Medical Academy, Zabrze, Poland), P. Rudnai (National Institute of Public Health, Budapest, Hungary), S. Skerfving (Dept of Occupational and Environmental Medicine, University Hospital, Lund Sweden), V. Vondra (Pulmonary Dept, Faculty Hospital, Prague, Czech Republic), D.W. Dockery and J. Schwartz (Dept of Environmental Health, Harvard School of Public Health, Boston, USA), J.P. Schouten (Dept of Epidemiology and Statistics, University of Groningen, The Netherlands), G. Baldini (Paediatric Clinic, University of Pisa, Italy), J. Clench-Aas (Norwegian Institute for Air Research Kjeller, Norway), N. Englert (Federal Environmental Agency, Berlin, Germany), P. Fischer (National Institute of Public Health and the Environment, Bilthoven, The Netherlands), B. Forsberg (Dept of Environmental Health, Umeå University, Sweden). 
$\mathrm{SO}_{2}$ and particulate levels and air pollution characterized by high levels of $\mathrm{NO}_{2}$ and diesel soot is of specific interest. East European countries still experience the classical type of air pollution, whereas west European countries experience more motor vehicle air pollution. A comparison of health effects between urban areas and suburban or rural areas is of interest to detect the effects of primary urban emissions.

The Pollution Effects on Asthmatic Children in Europe (PEACE) study was designed to study the relationship between short-term changes in air pollution and lung function, respiratory symptoms and medication use. It is a collection of panel studies that were conducted in the winter of 1993/1994 in 14 different centres in Europe. All centres used the same protocol for data collection and data analysis. The design, methods and results of the individual panels have been reported in a special issue of the European Respiratory Review [8]. In this paper, the effect estimates on respiratory health for the separate panels are used to calculate combined effect estimates for various air pollutants using meta-analysis techniques. In addition to combining effect estimates of all locations, differences between locations based on geographic location, urban/suburban location and composition of air pollution mixture were also investigated. The evaluation of differences in response between subjects within a panel, such as asthmatics versus nonasthmatics and atopics versus nonatopics, will be the topic of a separate paper.

\section{Materials and methods}

\section{PEACE study}

The PEACE study was a collaboration of 14 European centres: Amsterdam (The Netherlands), Kuopio (Finland), Oslo (Norway), Berlin and Hettstedt (Germany), Pisa (Italy), Athens (Greece), Kraków and Katowice (Poland), Prague and Teplice (Czech Republic), Budapest (Hungary) and Umeå and Malmö (Sweden). Each centre selected two panels, one in an urban area and one in a suburban or rural area (hereafter referred to as the suburban panel). The suburban panel was selected from a community which had no major traffic emissions, had no large industrial sources, had sufficient size to select enough subjects and was close to a site of an existing air pollution measurement network. Suburban panels were included to evaluate differences in the effects of air pollution caused by level and composition of air pollution, in panels paired by meteorological characteristics.

Children aged between 6 and 12 yrs with chronic respiratory symptoms were selected by a parent-completed screening questionnaire. The criteria for selection were: reporting of wheeze (apart from colds), attacks of shortness of breath with wheezing, and/or dry cough (apart from colds) in the last 12 months and/or doctor-diagnosed asthma ever in life. To characterize the children further, skin-prick tests to common allergens were applied, lung function was measured and a detailed questionnaire on housing characteristics, environmental tobacco smoke (ETS) exposure and parental education was administered to the parents. The methods are given in detail elsewhere [8].
Peak expiratory flow (PEF) was measured each day in the morning and in the evening for at least 2 months in the winter of 1993/1994. Winter was chosen as the study season because in Europe the highest particle and $\mathrm{SO}_{2}$ concentrations usually occur in winter. The starting date in each location was chosen such that the highest air pollution levels during winter were expected to occur during the measurement period and the pollen season would not have started. Starting dates ranged from November 1993 in Hettstedt, Germany, to February 1994 in Kuopio, Finland. More detailed information is given elsewhere [8]. Three PEF measurements were performed in a standing position, prior to medication use. All three readings were noted in a diary. The highest of the three readings was used for analysis. All subjects used the miniWright peak flow meter (Clement Clarke, Essex, UK). A parent completed a daily diary for the child, recording the presence and severity of respiratory symptoms and use of medication for respiratory symptoms. The symptoms in the diary were cough, phlegm, runny/stuffed nose, woken up with breathing problems, shortness of breath, wheeze, attack(s) of shortness of breath with wheeze, fever, eye irritation and sore throat. To avoid large changes in composition of the reporting group of children on separate days, children were included in the analysis if they had valid PEF measurements and respiratory symptom data on $>60 \%$ of the days.

Concurrent air pollution measurements were performed in both the urban and suburban locations. Daily 24-h measurements of PM10, $\mathrm{BS}, \mathrm{SO}_{2}$ and $\mathrm{NO}_{2}$ were made at sites not influenced by nearby sources, i.e. background sites. More information on the measurement methods is given elsewhere [8-10].

All panels were analysed separately. The first 2 days of each subject's data were removed to eliminate a possible training effect. Individual daily PEF readings were transformed into a daily population variable representing the population mean for each day of the individual deviations from the child-specific mean PEF [1, 2]. This was done separately for morning and evening PEF, resulting in $\triangle \mathrm{PEF}_{\mathrm{am}}$ and $\triangle \mathrm{PEF}_{\mathrm{pm}}$. The association between daily levels of air pollutants and daily levels in $\triangle \mathrm{PEF}_{\mathrm{am}}$ and $\triangle \mathrm{PEF}_{\mathrm{pm}}$ was calculated by means of linear regression weighted by the number of reporting children on each day. Correction for autocorrelation in the residuals was made. A firstorder autoregressive model was identified after inspection of the partial autocorrelation function of the residuals.

The symptoms in the diaries were recoded to 0 (no symptom) and 1 (slight, moderate or severe symptom) and the daily prevalence was calculated. Daily prevalence was defined as the fraction of children for whom the presence of a respiratory symptom/medication use was reported from those children providing valid diary data for that symptom on that day. Daily prevalence was preferred above daily incidence in order to obtain comparable results with earlier studies [1-4]. The association between symptom prevalence and air pollution was evaluated with logistic regression but under the assumption of normally distributed residuals. This was done because when analysing prevalence with binomial distributed residuals the residuals showed significant underdispersion. The observations were weighted by the number of reporting children on each day and correction for first-order autocorrelation in the residuals was made after inspection of the residuals. 
The respiratory symptoms cough and phlegm and the symptom combinations upper respiratory symptoms (runny/stuffed nose, sore throat) and lower respiratory symptoms (shortness of breath, wheeze, asthma attacks) were analysed. The prevalence of bronchodilator use (e.g. salbutamol (albuterol), fenoterol or terbutaline) was also analysed.

The explanatory variables were $24 \mathrm{~h}$ average concentrations of PM10, $\mathrm{BS}, \mathrm{SO}_{2}$ and $\mathrm{NO}_{2}$, analysed separately because of the high correlation $(r>0.6)$ between the pollutants. Current-day concentration (lag0), previous-day concentration (lag1), concentration 2 days before (lag2) and the average of lag 0-6 days (7 day mean) were analysed separately. The choice of these lags was based primarily on findings in previous studies [1-3]. Minimum temperature, a dummy variable indicating normal school days versus holidays/weekends and time trend were included as potential confounders. Time trend was included as a linear and square-root term for the PEF analysis and as a thirdorder polynomial in the prevalence analysis. The regression slopes of the air-pollution components from the linear and logistic regression models for each panel were used to calculate combined effect estimates.

\section{Statistical methods and analysis}

A combined analysis of panel-specific effect estimates using meta-analysis techniques was chosen over a pooled analysis because of the computational complexity of the latter. A pooled analysis, involving the creation of one large data set of all individual data, would require interaction terms for each term included in the regression equation to allow for, for example, panel-specific time trends. Combined effect estimates were calculated for the regression slopes of lag0, lag1, lag2, and the 7 day mean on $\triangle \mathrm{PEF}_{\mathrm{am}}, \triangle \mathrm{PEF}_{\mathrm{pm}}$ and the prevalence of respiratory symptoms and bronchodilator use. For PEF, the effect is expressed as $\mathrm{L} \cdot \mathrm{min}^{-1}$ change in PEF, whereas for prevalence the effect is expressed as an odds ratio (OR), both per 100 $\mu \mathrm{g} \cdot \mathrm{m}^{-3}$ increase in air pollution. A combined fixed effect estimate was calculated as the weighted mean of the panelspecific slopes with the weights inversely proportional to the panel-specific variance. The standard error (SE) of the combined slope was calculated as the inverse of the square root of the sum of the weights [11]. This fixed effect mean assumes that the variability in panel-specific slopes is caused by sampling errors only and that there is no variance present caused by other factors. Panel-specific regression slopes and the combined regression slope were plotted with $95 \%$ confidence intervals (CI). Heterogeneity of panel-specific slopes was evaluated by a visual inspection followed by a Chi-squared test for homogeneity [11]. In this visual inspection, heterogeneity was suspected when the combined slope was not contained in the $95 \%$ CI of all panel-specific slopes [12]. In the case of homogeneity, the combined slope calculated as a fixed effect was considered an appropriate estimate. A conservative cut-off point of a p-value smaller than 0.25 was chosen to determine heterogeneity. In case of heterogeneity $(p<0.25)$, combined effect estimates using random effect estimation were calculated with the noniterative method with unequal weights [11]. Random effect estimation takes into account both the within-study and the between-study variance.
Next, combined effect estimates were calculated within predefined strata. Location (urban versus suburban) was used to evaluate the potential additional effect of urban air pollution sources over suburban areas. Geographical location was used to evaluate climatic influences or regional differences in air pollution. Four groups were defined: north (Umeå, Oslo, Malmö, Kuopio), west (Amsterdam, Berlin), east (Hettstedt, Kraków, Katowice, Teplice, Prague, Budapest) and south (Pisa, Athens). Strata based on concentrations of air pollution components were defined to evaluate possible modification of effects by the composition of air pollution. BS measures the reflectance of small particles and served as an indicator of fine black carbon particles emitted by traffic or coal combustion (soot). $\mathrm{SO}_{2}$ served as an indicator of air pollution caused by fossil fuel combustion with high amounts of sulphur such as certain types of coal and oil. $\mathrm{NO}_{2}$ served as an indicator of traffic-related air pollution, since motor vehicles emit large amounts of $\mathrm{NO}$ which rapidly reacts to $\mathrm{NO}_{2}$. Strata based on the ratio between the mean concentrations of PM10 and BS served to indicate the proportion of carbonaceous particles within PM10.

To correct for other factors, a weighted multiple linear regression was performed with the panel-specific regression slopes, with the inverse of the panel-specific variance of the slope used as weights. The calculated SE of the regression slope was corrected according to BERLIN et al. [13]. Separate independent variables were mean concentrations of PM10, BS, $\mathrm{SO}_{2}$ and $\mathrm{NO}_{2}$, the ratio $\mathrm{PM} 10 / \mathrm{BS}$ and geographical position. To evaluate unmeasured differences between urban and suburban locations, a dummy indicator for location was included in the regression models. Children who were selected only on the basis of a positive answer to the nightly dry cough question had a lower prevalence of lower respiratory symptoms, upper respiratory symptoms and phlegm than children selected on asthma symptoms [14] and reacted differently to air pollution in the Finnish panels [15]. Thus, the reaction of a panel to air pollution might be influenced by panel composition. This may affect the relationship between the regression slopes and indicators of air pollution composition. The percentage of atopic children, the percentage of children in a panel who were selected only on the basis of a positive answer to cough and the mean prevalence of bronchodilator use of a panel served as indicators of panel composition and were, therefore, included in the regression models to adjust the results. This paper will focus only on indicators of air pollution composition as possible effect modifiers. A formal analysis of subgroups within the PEACE panels, such as atopic children, children using respiratory medication or children included on different selection questions, will not be discussed in this paper and will be the subject of a separate paper.

\section{Results}

A total of 66,879 questionnaires was handed out: $51,786(77 \%)$ were returned and 8,308 (16\%) children fulfilled the selection criteria. The design called for 75 children in each panel, i.e. a total of 2,100 children in 28 locations. From the 2,371 children who were enrolled, 2,010 were included in the analysis. The children included in the analysis did not differ from the excluded children 
Table 1. - Characteristics of the (Pollution Effects on Asthmatic Children in Europe) panels

\begin{tabular}{|c|c|c|c|c|c|c|c|c|c|c|}
\hline & & $\begin{array}{l}\mathrm{PM} 10^{*} \\
\mu \mathrm{g} \cdot \mathrm{m}^{-3}\end{array}$ & $\begin{array}{c}\mathrm{BS}^{*} \\
\mu \mathrm{g} \cdot \mathrm{m}^{-3}\end{array}$ & $\begin{array}{c}\text { PM10/ } \\
\text { BS ratio }\end{array}$ & $\begin{array}{c}\mathrm{SO}_{2}^{*} \\
\mu \mathrm{g} \cdot \mathrm{m}^{-3}\end{array}$ & $\begin{array}{l}\mathrm{NO}_{2}^{*} \\
\mu \mathrm{g} \cdot \mathrm{m}^{-3}\end{array}$ & $\begin{array}{c}\text { Subjects } \\
n\end{array}$ & Atopic $_{\%}^{\ddagger}$ & Cough $_{\%}^{\#}$ & $\begin{array}{l}\text { Bron }{ }^{\S} \\
\%\end{array}$ \\
\hline$\overline{\text { Umeå }}$ & Urban & 13.4 & 4.6 & 2.9 & 2.7 & 25.0 & 75 & 54 & 15 & 10 \\
\hline (Sweden) & Suburban & 11.5 & 5.3 & 2.2 & 4.0 & 15.3 & 72 & 61 & 17 & 22 \\
\hline Malmö & Urban & 22.9 & 8.2 & 2.8 & 6.0 & 20.7 & 78 & 50 & 28 & 18 \\
\hline (Sweden) & Suburban & 16.2 & 4.5 & 3.6 & 4.0 & 8.9 & 82 & 55 & 34 & 8 \\
\hline Kuopio & Urban & 17.7 & 12.6 & 1.4 & 6.0 & 28.4 & 85 & 64 & 54 & 3 \\
\hline (Finland) & Suburban & 13.0 & 7.9 & 1.6 & - & 13.7 & 84 & 64 & 58 & 7 \\
\hline Oslo & Urban & 19.3 & 27.6 & 0.7 & 12.4 & 49.3 & 56 & 41 & 36 & 3 \\
\hline (Norway) & Suburban & 11.2 & 13.1 & 0.9 & 3.4 & 15.3 & 68 & 49 & 55 & 10 \\
\hline Amsterdam & Urban & 45.3 & 16.5 & 2.7 & 13.2 & 46.4 & 55 & 51 & 38 & 4 \\
\hline $\begin{array}{l}\text { (The } \\
\text { Netherlands) }\end{array}$ & Suburban & 44.4 & 13.6 & 6.0 & 8.5 & 26.5 & 71 & 41 & 44 & 3 \\
\hline Berlin & Urban & 52.3 & 24.5 & 2.1 & 42.3 & 38.3 & 50 & 60 & 4 & 17 \\
\hline (Germany) & Suburban & 43.0 & 22.0 & 2.0 & 26.1 & 21.2 & 66 & 59 & 3 & 8 \\
\hline Hettstedt & Urban & 40.3 & 42.0 & 1.0 & 83.3 & 26.5 & 75 & 33 & 21 & 2 \\
\hline (Germany) & Suburban & 32.9 & 25.5 & 1.3 & 64.9 & 26.1 & 63 & 19 & 10 & 5 \\
\hline Katowice & Urban & 68.7 & 55.5 & 1.2 & 55.7 & 68.7 & 72 & 49 & 35 & 1 \\
\hline (Poland) & Suburban & 73.8 & 57.9 & 1.3 & 56.0 & 69.5 & 73 & 30 & 15 & 3 \\
\hline Kraków & Urban & 60.1 & 34.9 & 1.7 & 41.3 & - & 73 & 7 & 51 & 1 \\
\hline (Poland) & Suburban & 56.1 & 42.7 & 1.3 & 14.0 & - & 76 & 53 & 31 & 1 \\
\hline Teplice & Urban & 74.3 & 58.9 & 1.3 & 74.8 & 48.8 & 91 & 16 & 56 & 3 \\
\hline $\begin{array}{l}\text { (Czech } \\
\text { Republic) }\end{array}$ & Suburban & 32.4 & 22.0 & 1.5 & 19.9 & 12.5 & 77 & 26 & 36 & 1 \\
\hline Prague & Urban & 52.7 & 29.4 & 1.8 & 113.9 & 44.7 & 66 & 48 & 2 & 5 \\
\hline $\begin{array}{l}\text { (Czech } \\
\text { Republic) }\end{array}$ & Suburban & 49.6 & 20.8 & 2.4 & 30.8 & 12.9 & 68 & 81 & 14 & 20 \\
\hline Budapest & Urban & 60.9 & 48.9 & 1.2 & 49.7 & 35.3 & 76 & 40 & 45 & 2 \\
\hline (Hungary) & Suburban & 52.1 & 30.6 & 1.7 & 41.0 & 25.4 & 63 & 58 & 33 & 5 \\
\hline Pisa & Urban & 61.6 & 19.7 & 3.1 & 15.7 & 68.1 & 68 & 81 & 0 & 4 \\
\hline (Italy) & Suburban & 69.5 & 29.3 & 2.4 & 8.2 & 32.7 & 60 & 59 & 19 & 6 \\
\hline Athens & Urban & 98.8 & 109.2 & 0.9 & 72.4 & 74.9 & 87 & 16 & 53 & 4 \\
\hline (Greece) & Suburban & 50.0 & 33.5 & 1.5 & - & 19.7 & 80 & 22 & 31 & 7 \\
\hline
\end{tabular}

*: mean concentration during the study period; $\uparrow$ : ratio of mean concentrations; positive skin-prick test reactions; \#: percentage of children in panel selected only on basis of question on nightly coughing; $\$$ : mean prevalence of bronchodilator (Bron.) use in panel during the study period. PM10: particles with a 50\% cut-off aerodynamic diameter of $10 \mu \mathrm{m}$; BS: black smoke.

with respect to responses on screening questions, skinprick testing or lung function levels. The most important characteristics of the panels and results of air pollution measurements are summarized in table 1 . A wide range of air pollution concentrations were included, with low concentrations of both gaseous and particle components in northern Europe, higher concentrations in western Europe and the highest concentrations in central and southern Europe. The ratio between the mean concentration of PM10 and BS varied widely between sites, but did not show a geographical pattern. More details on air pollution have been published by НоЕк et al. [9].

The weighted means of the panel-specific estimates of the air pollutants on $\triangle \mathrm{PEF}_{\mathrm{am}}$ and $\triangle \mathrm{PEF}_{\mathrm{pm}}$ are presented in table 2. PM10 and BS measurements were performed in all 28 locations. No $\mathrm{SO}_{2}$ measurements were taken in Kuopio suburban location or Athens suburban location and no $\mathrm{NO}_{2}$ measurements were taken in Kraków urban and suburban locations. For these components, 26 panel-specific regression slopes were available for each representation. Heterogeneity was predominantly present in the effect estimates of the 7 day means of the air pollution components. Most combined effect estimates were positive, but mostly nonsignificant. A positive association means that an increase in air pollution is associated with an increase in PEF, which is opposite to the expected asso- ciation. A significant positive association was found between $\mathrm{SO}_{2}$ lag2 and $\triangle \mathrm{PEF}_{\mathrm{am}}$. The only significant negative association was found for PM10 lag1 with $\triangle \mathrm{PEF}_{\mathrm{pm}}$.

The OR calculated from the combined effect estimates of the air pollutants on symptom prevalence and bronchodilator use are presented in table 3 . In addition to the missing air pollution data in the above-mentioned locations, the prevalence of bronchodilator use was very low and showed little variation in Katowice urban location, and Kraków suburban location, which resulted in nonconverging models. Most combined OR were below 1.00, with significant $\mathrm{OR}$ for $\mathrm{PM} 10$ and $\mathrm{SO}_{2} \operatorname{lag} 0$ on cough prevalence and $\mathrm{PM} 10, \operatorname{lag} 1$ and 7 day mean, BS 7 day mean and $\mathrm{SO}_{2}$ lag 2 on the prevalence of upper respiratory symptoms. An $\mathrm{OR}<1$ means that an increase in air pollution was associated with a decrease in prevalence, which is opposite to the expected association. Heterogeneity was present for all effect estimates of all components on all symptoms and bronchodilator use, but most consistently for 7 day mean effect estimates.

Stratification according to urban or suburban location of the effect estimates of PM10 lag1 on $\Delta \mathrm{PEF}_{\mathrm{pm}}$ and on the prevalence of upper respiratory symptoms, lower respiratory symptoms and bronchodilator use are presented in table 4. PM10 lag1 estimates and upper respiratory symptoms, lower respiratory symptoms and bronchodilator 
Table 2. - Combined effect estimates with 95\% confidence intervals $(\mathrm{Cl})$ of air pollution on peak expiratory flow (PEF)

\begin{tabular}{|c|c|c|c|}
\hline & $\mathrm{n}^{*}$ & $\begin{array}{c}\Delta \mathrm{PEF}_{\mathrm{am}} \\
\text { Mean }(95 \% \mathrm{CI})\end{array}$ & $\begin{array}{c}\Delta \mathrm{PEF}_{\mathrm{pm}} \\
\text { Mean }(95 \% \mathrm{CI})\end{array}$ \\
\hline \multicolumn{4}{|l|}{ PM10 } \\
\hline lag0 & 28 & $0.5(-0.1,1.1)^{\dagger}$ & $0.4(-0.1,0.9) \dagger$ \\
\hline lag1 & 28 & $0.1(-0.5,0.7)$ & $-0.6(-1.1,-0.1)$ \\
\hline lag2 & 28 & $0.5(-0.1,1.1)^{\dagger}$ & $0.2(-0.5,0.9)$ \\
\hline 7 day mean & 28 & $0.2(-1.6,2.0)$ & $0.0(-1.7,1.7)$ \\
\hline \multicolumn{4}{|l|}{ BS } \\
\hline $\operatorname{lag} 0$ & 28 & $0.5(-0.1,1.1)$ & $0.1(-0.5,0.7) \dagger$ \\
\hline lag1 & 28 & $-0.1(-0.9,0.7)$ & $-0.3(-0.9,0.3)^{\dagger}$ \\
\hline $\operatorname{lag} 2$ & 28 & $0.3(-0.6,1.2)$ & $0.4(-0.2,1.0) \dagger$ \\
\hline 7 day mean & 28 & $0.9(-1.6,3.4)$ & $0.6(-1.5,2.7)$ \\
\hline \multicolumn{4}{|l|}{$\mathrm{SO}_{2}$} \\
\hline $\operatorname{lag} 0$ & 26 & $0.2(-0.2,0.6) \dagger$ & $0.1(-0.3,0.5)^{\dagger}$ \\
\hline lag1 & 26 & $0.2(-0.2,0.6)$ & $0.0(-0.4,0.4)^{\dagger}$ \\
\hline $\operatorname{lag} 2$ & 26 & $0.6(0.2,1.0)^{\dagger}$ & $0.1(-0.4,0.6) \dagger$ \\
\hline 7 day mean & 26 & $0.6(-1.3,2.5)$ & $0.2(-0.5,0.9)$ \\
\hline \multicolumn{4}{|l|}{$\mathrm{NO}_{2}$} \\
\hline $\operatorname{lag} 0$ & 26 & $0.7(-0.2,1.6)$ & $0.4(-0.5,1.3)$ \\
\hline lag1 & 26 & $1.1(-0.1,2.3)$ & $0.0(-0.9,0.9)$ \\
\hline lag2 & 26 & $0.6(-0.5,1.7)$ & $0.2(-0.7,1.1)$ \\
\hline 7 day mean & 26 & $0.2(-3.0,3.4)$ & $0.6(-3.1,4.3)$ \\
\hline
\end{tabular}

Data are expressed in L.min ${ }^{-1} \cdot 100 \mu \mathrm{g}^{-1} \cdot \mathrm{m}^{3} . *$ : number of panelspecific estimates; $;$ : fixed effects model; model. $\triangle \mathrm{PEF}_{\mathrm{am}}, \triangle \mathrm{PEF}_{\mathrm{pm}}$ : population mean of the individual deviations from the child-specific mean of morning and evening PEF, respectively; lag0: current-day concentration; lag1: previous-day concentration; lag2: concentration 2 days before; 7 day mean: average of lag 0-6 days; PM10: particles with a 50\% cutoff aerodynamic diameter of $10 \mu \mathrm{m}$; BS: black smoke.

use are presented because these have been reported frequently in the literature. No clear difference could be detected between the effect estimates in urban and suburban locations. This was also the case for $\triangle \mathrm{PEF}_{\mathrm{am}}$ and the prevalence of cough and phlegm. Lag0, lag2 and 7 day mean of PM10 and the other air pollutants and their representations did not show clear differences. Multiple regression analysis did not show a clear difference between the effect estimates in urban and suburban locations (data not shown).

Stratification according to geographical location in Europe (north, west, east, south) to evaluate climatic influences or regional differences in air pollution did not show consistent differences among locations for the effect estimates on $\triangle \mathrm{PEF}_{\mathrm{am}}$ and $\triangle \mathrm{PEF}_{\mathrm{pm}}$. As an example, PM10 lag1 and $\triangle \mathrm{PEF}_{\mathrm{pm}}$ are shown in table 4 . The effect estimates on the prevalence of upper respiratory symptoms, lower respiratory symptoms and bronchodilator use are also shown. In general, the effect estimates on symptom prevalence in the east stratum had the smallest confidence intervals, probably caused by the large range in air pollution concentrations and the relatively large number of locations included. The north stratum had, for PM10 and BS, the highest OR for the respiratory symptoms cough, phlegm, upper respiratory symptoms and lower respiratory symptoms. Most of these were insignificant except for BS lag1 on cough prevalence, namely 1.26 (95\% CI 1.04-1.52). Further, some significant OR in the unexpected direction could be seen in the east and west strata. The stratum-specific OR for the 7 day mean concentrations were significant in the south stratum for cough with BS 7 day mean (1.44; 95\% CI 1.06-1.94) and $\mathrm{SO}_{2} 7$ day mean (2.26; 95\% CI 1.30-3.92) and for phlegm with $\mathrm{SO}_{2} 7$ day mean $(3.24 ; 95 \%$ CI 1.83-5.75). None of the other combined effect es-timates for the other representations, air pollutants or hea-lth outcomes showed a clear pattern. Heterogeneity was still present within the strata. The multiple regression analysis with the geographical locations included as dummies re-sulted in mostly insignificant differences between the loca-tions and there was no consistent pattern (data not shown).

Stratification in tertiles of the ratio between the mean concentration of PM10 and BS showed that most of the combined effect estimates of PM10 on $\triangle \mathrm{PEF}_{\mathrm{pm}}$ in the low stratum were negative and in the medium and high strata predominantly positive (table 5). This was also true for effect estimates of BS (data not shown). Effect estimates for lag1 of PM10 and BS became more positive with increasing ratio. Adjusted regression analysis with the PM10/ $\mathrm{BS}$ ratio as a continuous variable showed that this trend was borderline significant $(p<0.10)$ for the effect estimates of BS lag1 and of the 7 day means of PM10 and BS. Significant positive combined effect estimates were present in the medium stratum for lag2 and the 7 day means of PM10, significant negative effect estimates for PM10 lag1 in the low stratum (table 5). BS also showed significant positive combined effect estimates for lag2 and 7 day mean in the medium stratum (data not shown). Effect estimates of PM10 and BS on $\triangle \mathrm{PEF}_{\mathrm{am}}$ and effect estimates of $\mathrm{SO}_{2}$ and $\mathrm{NO}_{2}$ on both $\triangle \mathrm{PEF}_{\mathrm{am}}$ and $\Delta \mathrm{PEF}_{\mathrm{pm}}$ were not significantly related to $\mathrm{PM} 10 / \mathrm{BS}$ ratios (data not shown).

Stratification on the PM10/BS ratio of the effect estimates of PM10 lag1 and 7 day mean on the prevalence of upper respiratory symptoms, lower respiratory symptoms and bronchodilator use did not show a clear pattern (table 5). Lag1 effect estimates are presented because these have been reported most frequently in the literature and 7 day mean effect estimates because these showed most heterogeneity. Stratification on the PM10/BS ratio showed some pattern for cough and phlegm. More specifically, in the low stratum, the OR for PM10 and BS on cough and phlegm prevalence were above 1.00 for lag1 and 7 day mean, with the OR for BS 7 day mean on cough prevalence becoming significant $(1.29 ; 95 \%$ CI 1.02-1.64). In the other strata the OR were mostly below 1.00 (data not shown). The effect estimates of the other representations and other air pollutants were not related to the PM10/BS ratio. There was no clear trend in the multiple regression analysis with the PM10/BS ratio as a continuous variable for all symptoms and air pollutants.

Stratification of the effect estimates on $\triangle \mathrm{PEF}_{\mathrm{am}}, \Delta \mathrm{PEF}_{\mathrm{pm}}$ and prevalence of symptoms and bronchodilator use on the basis of mean concentration of $\mathrm{PM}_{10}, \mathrm{NO}_{2}$ or $\mathrm{SO}_{2}$ did not show obvious patterns (data not shown). Stratification of $\triangle \mathrm{PEF}_{\mathrm{pm}}$ effect estimates on tertiles of mean BS concentration showed negative effect estimates for PM10 lag1 in the highest tertile, but this trend was insignificant (data not shown).

\section{Discussion}

The combination of the panel-specific estimates into an aggregate estimate did not show a clear effect of air pollution on either PEF or the daily prevalence of respiratory 
Table 3. - Combined odds ratio (OR) and 95\% confidence intervals $(\mathrm{Cl})$ for a $100 \mu \mathrm{g} \cdot \mathrm{m}^{-3}$ increase in air pollution on symptom prevalence and bronchodilator use

\begin{tabular}{|c|c|c|c|c|c|c|c|c|c|c|}
\hline & \multicolumn{2}{|r|}{ Cough } & \multicolumn{2}{|r|}{ Phlegm } & \multicolumn{2}{|r|}{ URS } & \multicolumn{2}{|r|}{ LRS } & \multicolumn{2}{|c|}{ Bronchodilator use } \\
\hline & $\mathrm{n}^{*}$ & OR $(95 \% \mathrm{CI})$ & $\mathrm{n}^{*}$ & $5 \% \mathrm{CI})$ & $\mathrm{n}^{*}$ & OR $(95 \% \mathrm{CI})$ & $\mathrm{n}^{*}$ & OR $(95 \% \mathrm{CI})$ & n* & OR $(95 \% \mathrm{CI})$ \\
\hline \multicolumn{11}{|l|}{ PM10 } \\
\hline $\operatorname{go} 0$ & & $96(0.92,0.99)^{\dagger}$ & 0 & $.99(0.92,1.05)$ & 28 & $.97(0.94,1.01)^{\dagger}$ & 28 & $0.93(0.85,1.01)$ & 25 & $0.99(0.9$ \\
\hline lag1 & & ) & & 1) & 28 & $9)$ & 28 & .00 & 25 & \\
\hline lag2 & & $3)^{\dagger}$ & 28 & $5)^{\ddagger}$ & 28 & ) & 28 & .94 & 25 & $.07)^{\ddagger}$ \\
\hline 7 day mean & 28 & 0.90( & 28 & 0.89( & 28 & .81( & 28 & 0.88( & 25 & $.10)^{\ddagger}$ \\
\hline \multicolumn{11}{|l|}{ BS } \\
\hline lag0 & & or & & , & 28 & 0 & 2 & 02 & 25 & $1.06)^{\dagger}-(-1-$ \\
\hline lag1 & & & & & 28 & & 28 & & 25 & \\
\hline lag2 & & 0.99 & 28 & 0.97 & 28 & ) & 28 & 0.93 & 25 & $.05)^{\dagger}$ \\
\hline 7 day & 28 & $0.95(0.75,1.21)$ & 28 & $0.90(0.63$ & 28 & $0.81(0.7$ & 28 & 0.90( & 25 & $0.88(0.60,1.29)$ \\
\hline \multicolumn{11}{|l|}{$\mathrm{SO}_{2}$} \\
\hline lag0 & & & & & 0 & & 2 & 0.06 & 23 & \\
\hline lag1 & & & & & 26 & & 26 & & 23 & $1)^{\ddagger}$ \\
\hline lag2 & 26 & 0.94 & 26 & 0.96 & 26 & & 26 & 0.90 & 23 & $(1.08)$ \\
\hline 7 day mean & 26 & $0.84(0.69,1.03)$ & 26 & $0.80(0.60,1.06)$ & 26 & $0.83(0.69,1.00)$ & 26 & $0.88(0.58,1.33)$ & 23 & $0.88(0.68,1.13)$ \\
\hline \multicolumn{11}{|l|}{$\mathrm{NO}_{2}$} \\
\hline & & & & & 26 & & & & 24 & \\
\hline & & & & & 2 & & 2 & & 24 & 10 \\
\hline lag2 & 2 & 0.94( & 26 & 0.97 & 26 & 0.9 & 26 & $0.86(0$ & 24 & $0.99(0.89,1.10)^{\dagger}$ \\
\hline 7 day mean & 26 & $0.98(0.72,1.34)$ & 26 & $0.79(0.55,1.15)$ & 26 & $0.82(0.59,1.14)$ & 26 & $0.89(0.48,1.67)$ & 24 & $0.82(0.52,1.30)$ \\
\hline
\end{tabular}

*: number of panel-specific estimates. ${ }^{*}$ : fixed effects model; $*$ : random effects model. URS: upper respiratory symptoms; LRS: lower respiratory symptoms; lag0: current-day concentration; lag1: previous-day concentration; lag2: concentration 2 days before; 7 day mean: average of lag 0-6 days; PM10: particles with a 50\% cut-off aerodynamic diameter of $10 \mu \mathrm{m}$; BS: black smoke.

symptoms or bronchodilator use. The only significant negative association was PM10 lag1 with evening PEF. Earlier studies in general population samples of children showed an effect of winter air pollution on lung function [16, 17], but not on respiratory symptoms $[4,16,17]$. Other studies on winter air pollution in selected children showed a relation between air pollution and respiratory symptom prevalence [1-3], bronchodilator use [1, 3] and lung function [1-4]. These studies were mostly conducted in children with chronic respiratory symptoms. The lack of effect in this study cannot be explained by low levels of exposure. In all but the northern European locations, the air pollution concentrations were similar to or even higher than the levels in the studies mentioned above which showed effects. The statistical power was also sufficient. In the case of the daily prevalence of bronchodilator use or lower respiratory symptoms and a $10 \mu \mathrm{g} \cdot \mathrm{m}^{-3}$ increase in PM10 lag1, an OR of 1.01 would have reached statistical significance at the $5 \%$ probability level. In addition, the same increase in PM10 would have to be related to a reduction in PEF of only $0.06 \mathrm{~L} \cdot \mathrm{min}^{-1}$ to become significant at the $5 \%$ probability level. In relative terms, this is a reduction of $0.02 \%$, assuming a mean PEF of $300 \mathrm{~L} \cdot \mathrm{min}^{-1}$. These are much smaller effects than those that were calculated in a review using data from earlier panel studies [18]. Other panel studies have analysed the incidence of respiratory symptoms instead of prevalence [19]. Incidence data are affected less by autocorrelation. Daily incidence was also analysed in this study but, because of the low incidence of respiratory symptoms within the panels, the power was lower than the prevalence data. For example, the combined effect estimates of PM10 lag1 were 0.91 (95\% CI 0.78-1.07) on cough, 1.11 (95\% CI 0.91-1.35) on phlegm, 0.88 (95\% CI 0.761.02) on upper respiratory symptoms, 1.04 (95\% CI $0.85-1.27)$ on lower respiratory symptoms and $1.14(95 \%$ CI 0.80-1.64) on the incidence of bronchodilator use. In addition, since there were no clear effects on incidence and in order to be able to compare the results with most earlier panel studies, a choice was made to present prevalence analysis.

Table 4. - Combined effect estimates with $95 \%$ confidence intervals $(\mathrm{Cl})$ of a $100 \mu \mathrm{g} \cdot \mathrm{m}^{-3}$ increase in particles with a $50 \%$ cut-off aerodynamic diameter of $10 \mu \mathrm{m}$ (PM10) lag1 on $\triangle \mathrm{PEFpm}\left(\mathrm{L} \cdot \mathrm{min}^{-1}\right)$ and symptom prevalence (odds ratio), stratified on urban/suburban and geographical location

\begin{tabular}{|c|c|c|c|c|c|c|c|c|}
\hline & $\mathrm{n}^{*}$ & $\triangle \mathrm{PEFpm}$ & $\mathrm{n}^{*}$ & LRS & $\mathrm{n}^{*}$ & URS & $\mathrm{n}^{*}$ & $\begin{array}{c}\text { Bronchodilator } \\
\text { use }\end{array}$ \\
\hline Urban & 14 & $-0.4(-1.1,0.3)^{\dagger}$ & 14 & $1.00(0.89,1.11)^{\grave{\dagger}}$ & 14 & $0.95(0.90,1.00)^{\dagger}$ & 14 & $0.95(0.85,1.06)$ \\
\hline Suburban & 14 & $-0.8(-1.6,0.0)^{\dagger}$ & 14 & $1.00(0.83,1.19)$ & 14 & $0.97(0.92,1.02)^{\dagger}$ & 14 & $0.92(0.80,1.05)^{\ddagger}$ \\
\hline North & 8 & $-0.7(-3.4,2.0)^{\dagger}$ & 8 & $1.11(0.60,2.07)$ & 8 & $1.00(0.83,1.20)^{\dagger}$ & 8 & $0.94(0.74,1.18)^{\dagger}$ \\
\hline West & 4 & $-0.3(-1.9,1.3)^{\dagger}$ & 4 & $0.96(0.71,1.30)$ & 4 & $0.91(0.82,1.02)^{\dagger}$ & 4 & $0.83(0.70,0.99)^{\dagger}$ \\
\hline East & 12 & $-0.7(-1.4,0.0)^{\dagger}$ & 12 & $1.04(0.94,1.14)^{\dagger}$ & 12 & $0.97(0.93,1.01)^{\dagger}$ & 10 & $0.89(0.77,1.03)$ \\
\hline South & 4 & $-0.6(-1.8,0.6)^{\dagger}$ & 4 & $0.91(0.81,1.02)$ & 4 & $0.94(0.87,1.02)^{\dagger}$ & 4 & $1.08(0.96,1.22)$ \\
\hline
\end{tabular}

North: Umeå, Malmö, Kuopio, Oslo; West: Amsterdam, Berlin; East: Hettstedt, Kraków, Katowice, Teplice, Prague, Budapest; South: Pisa, Athens. *: number of panel-specific estimates; $\uparrow$ : fixed effects model; $:$ : random effects model. lag1: previous-day concentration; $\triangle \mathrm{PEF}$ pm: population mean of the individual deviations from the child-specific mean evening peak expiratory flow; LRS: lower respiratory symptoms; URS: upper respiratory symptoms. 
Table 5. - Combined effect estimates with $95 \%$ confidence intervals $(\mathrm{Cl})$ of a $100 \mu \mathrm{g} \cdot \mathrm{m}^{-3}$ increase in particles with a $50 \%$ cut-off aerodynamic diameter of $10 \mu \mathrm{m}\left(\mathrm{PM}_{10}\right)$ on $\triangle \mathrm{PEFpm}\left(\mathrm{L} \cdot \mathrm{min}^{-1}\right)$ and symptom prevalence (odds ratio), stratified on tertiles of ratio $\mathrm{PM} 10$ and black smoke (BS)

\begin{tabular}{|c|c|c|c|c|c|c|}
\hline & \multicolumn{6}{|c|}{$\mathrm{PM} 10 / \mathrm{BS}$ ratio } \\
\hline & \multicolumn{2}{|r|}{ Low } & \multicolumn{2}{|r|}{ Medium } & \multicolumn{2}{|r|}{ High } \\
\hline & $\mathrm{n}^{*}$ & Mean $(95 \% \mathrm{CI})$ & $\mathrm{n}^{*}$ & Mean $(95 \% \mathrm{CI})$ & $\mathrm{n}^{*}$ & Mean $(95 \% \mathrm{CI})$ \\
\hline \multicolumn{7}{|l|}{$\overline{\mathrm{PEFpm}}$} \\
\hline $\operatorname{lag} 0$ & 9 & $0.2(-0.6,1.0)$ & 10 & $0.8(-0.1,1.7)^{\dagger}$ & 9 & $0.4(-0.8,1.6)^{\dagger}$ \\
\hline lag1 & 9 & $-0.9(-1.7,-0.1)$ & 10 & $-0.4(-1.3,0.5)$ & 9 & $-0.3(-1.5,0.9)^{\dagger}$ \\
\hline lag2 & 9 & $-0.7(-1.5,0.1)$ & 10 & $1.4(0.5,2.3)$ & 9 & $0.3(-0.9,1.5)$ \\
\hline 7 day mean & 9 & $-2.3(-5.0,0.4)$ & 10 & $2.5(0.8,4.2)^{\dagger}$ & 9 & $0.1(-4.3,4.5)$ \\
\hline \multicolumn{7}{|c|}{ Upper respiratory symptoms } \\
\hline lag1 & 9 & $0.96(0.91,1.02)^{\dagger}$ & 10 & $0.96(0.90,1.01)^{\dagger}$ & 9 & $0.96(0.89,1.03) \dagger$ \\
\hline 7 day mean & 9 & $0.90(0.65,1.23)$ & 10 & $0.75(0.63,0.90)$ & 9 & $0.79(0.59,1.05)$ \\
\hline \multicolumn{7}{|c|}{ Lower respiratory symptoms } \\
\hline lag1 & 9 & $0.99(0.89,1.09)^{\dagger}$ & 10 & $0.92(0.81,1.04) \dagger$ & 9 & $1.12(0.87,1.44)$ \\
\hline 7 day mean & 9 & $1.34(0.84,2.14)$ & 10 & $0.62(0.38,1.00)$ & 9 & $0.88(0.47,1.68)$ \\
\hline \multicolumn{7}{|c|}{ Bronchodilator use } \\
\hline $\operatorname{lag} 1$ & 8 & $1.01(0.91,1.12)^{\dagger}$ & 9 & $0.85(0.68,1.05)$ & 9 & $0.99(0.91,1.08) \dagger$ \\
\hline 7 day mean & 8 & $0.81(0.49,1.36)$ & 9 & $0.82(0.49,1.37)$ & 9 & $1.14(0.88,1.47) \dagger$ \\
\hline
\end{tabular}

*: number of panel-specific estimates; $\uparrow$ : fixed effects model; $:$ random effects model. $\triangle$ PEFpm: population mean of the individual deviations from the child-specific mean evening peak expiratory flow; low: ratio PM10/BS ð1.3; medium: 1.3 < ratio PM10/BS ð2.1; high: ratio PM10/BS >2.1; lag0: current-day concentration; lag1: previous-day concentration; lag2: concentration 2 days before; 7 day mean: average of lag 0-6 days.

The lack of effect probably cannot be attributed to incorrect specification of long-term time trends in the panel analysis. Misspecification of long-term time trends in respiratory symptom prevalence or PEF can bias the relation between acute effects and short-term changes in air pollution. A more detailed model was used to allow for training effects and/or unspecified time trends, compared with earlier panel studies $[1-3,5]$ in which linear trends or no trends were specified. Trends in the PEACE panels were, however, highly nonlinear. Residuals were checked and if a period longer than 14 days was found with positive or negative residuals this was corrected with dummy variables [8]. This procedure did not materially affect the effect estimates. In addition, for the PEF effect estimates two panels and for the symptom prevalence effect estimates nine panels were selected which made a large contribution to the combined effect estimate and had an effect estimate opposite to that expected. These panels were reanalysed using a nonparametric function of time to allow a more flexible relationship [20]. Specifically, loess was used with the span selected by Akaike's Information Criterion. When negative autocorrelation was present in the residuals, the span was increased. The effect estimates obtained with these models were similar to the effect estimates used here [8]. Acute respiratory infections are known to influence children's lung function [21] and episodes of respiratory infections may have biased the effect estimates on PEF as well as on symptom prevalence, depending on the association in time between respiratory infections and air pollution. Fever was recorded in the diaries of the subjects as a crude measure of infectious disease, but no sudden increases in the prevalence of fever were noted in any of the panels. Fever may not be sensitive enough to detect respiratory infections, but, unfortunately, no other data were available to evaluate the influence of respiratory infections for the PEACE study as a whole. However, data from a sentinel system available in The Netherlands suggested that influenza prevalence, as measured by the participating family doctors in the sentinel system, was a predictor of outcome variables in the Dutch panels. Furthermore, adjustment for these influenza prevalence data resulted in associations between air pollution and respiratory health which were generally in the expected direction of more air pollution leading to more cases of worse respiratory health (S.C. van der Zee, unpublished data). It is, therefore, possible that with more refined data on respiratory infections, the results may also be different in other locations. It is also possible that subgroups within the panels, such as asthmatics, nonmedication users or atopics, had a different response. This will be explored in a separate paper.

Tests on heterogeneity indicated that differences existed between some panel-specific estimates. These differences were larger in the respiratory symptoms analysis than in the analysis of the PEF data. The differences are unlikely to be caused by differences in study design, methods or data analysis because all centres used the same protocol for fieldwork and data analysis [8]. A probable cause for the larger heterogeneity might be linguistic and cultural differences in respiratory symptom reporting and medication use, although an attempt was made to eliminate this by backtranslating the diary forms. Factors which serve as an indicator of the composition of air pollution, such as mean concentrations of $\mathrm{PM} 10, \mathrm{SO}_{2}$ and $\mathrm{NO}_{2}$ were not able to explain the variation. $\mathrm{SO}_{2}$ served as an indicator of fossil fuel combustion with high sulphur amounts, whereas $\mathrm{NO}_{2}$ served as an indicator for traffic-related air pollution. By stratification on the mean level of $\mathrm{SO}_{2}$ and $\mathrm{NO}_{2}$, it was hoped that information could be obtained on the interaction between PM10 and these pollutants. Urban/suburban locations did not show differences between effect estimates. An explanation might be that the contrast between urban and suburban areas was not large with regard to levels of PM10 and BS [9]. Geographical location was not able to explain much variation, in contrast to another European multicentre study (Air Pollution and Health: a European Approach (APHEA)) in which the distinction between western and eastern European cities explained heterogeneity in the effect estimates of $\mathrm{BS}$ and $\mathrm{SO}_{2}$ on mortality [22].

In the PEACE study, the only significant negative association between air pollution and PEF was between PM10 lag 1 and $\triangle \mathrm{PEF}_{\mathrm{pm}}$ in centres with high $\mathrm{BS}$ concentra- tions 
or in centres with a low ratio between PM10 and BS. A similar, but insignificant, trend was present in the prevalence analysis. Removal of the centre with extreme BS concentrations (urban Athens) did not change these results. BS measures the reflectance of a sampled filter [23] and does not account for the portion of aerosol mass that does not absorb light [24]. The main fraction of lightabsorbing particles in ambient air is formed by elemental carbon [25] and the size of the particles sampled with the BS sampler is below $5 \mu \mathrm{m}$ [24]. The formulae used to transform reflectance to mass concentrations are based on older studies in which the air pollution mixture was different from that of today [25]. For this reason, the BS figures should be interpreted as an indication of elemental carbon and not as mass concentrations. Thus, there is a weak suggestion that particulate matter mixtures with a relatively high amount of elemental carbon, to which diesel and coal combustion contribute [6], were more likely to affect PEF than mixtures with low elemental carbon content. However, this is contradicted by the fact that in this study daily variations in BS were not related to variations in PEF or daily symptom prevalence.

Stratification of the associations between air pollution and $\triangle \mathrm{PEF}_{\mathrm{am}}$ did not show clear patterns. This might be explained by the timing of the morning PEF measurement. This was taken just after getting up, which means that a long period of indoor exposure preceded the measurement. This may have obscured a possible association of $\triangle \mathrm{PEF}_{\mathrm{am}}$ with outdoor air pollution.

The analysis of the PEACE data involved a large amount of statistical testing. This implies that the few associations that were significant might have been due to chance. By presenting all of the combined effect estimates (tables 2 and 3 ) and not only the significant associations in the expected direction, an attempt has been made to give an overview of the possible trends in the data, regardless of significance.

In conclusion, the Pollution Effects on Asthmatic Children in Europe project did not show clear effects of particles with a $50 \%$ cut-off aerodynamic diameter of $10 \mu \mathrm{m}$, black smoke, sulphur dioxide or nitrogen dioxide on morning or evening peak expiratory flow or the prevalence of respiratory symptoms and bronchodilator use. Only the previous-day concentration of particles with a 50\% cut-off aerodynamic diameter of $10 \mu \mathrm{m}$ was associated with evening peak expiratory flow, especially in locations where black smoke was high compared with the concentrations of these particles.

\section{References}

1. Pope CA III, Dockery DW, Spengler JD, Raizenne ME. Respiratory health and PM10 pollution: a daily time series analysis. Am Rev Respir Dis 1991; 144: 668-674.

2. Pope CA III, Dockery DW. Acute health effects of PM10 pollution on symptomatic and asymptomatic children. Am Rev Respir Dis 1992; 145: 1123-1128.

3. Roemer W, Hoek G, Brunekreef B. Effect of ambient winter air pollution on respiratory health of children with chronic respiratory symptoms. Am Rev Respir Dis 1993; 147: $118-124$

4. Hock G, Brunekreef B. Acute effects of a winter air pollution episode on pulmonary function and respiratory symptoms in children. Arch Environ Health 1993; 48: 328-335.

5. Peters A, Goldstein IF, Beyer U, et al. Acute health effects of exposure to high levels of air pollutants in Eastern Europe. Am J Epidemiol 1996; 144: 570-581.

6. World Health Organization. Air quality guidelines for Europe. Copenhagen, WHO Regional Publications, European Publications, European Series No. 23, 1987.

7. Katsouyanni K (ed.). Report Number 4: Study designs. Air pollution epidemiology reports series. Commission of the European Communities, Directorate-General XII for Science, Research and Development, EUR 15095 EN, 1995.

8. Roemer W, Hock G, Brunekreef B, et al. Effect of shortterm changes in urban air pollution on the respiratory health of children with chronic respiratory symptoms the PEACE project: Introduction. Eur Respir Rev 1998; 8: 4-11.

9. Hoek G, Forsberg B, Borowska M, et al. Wintertime PM10 and black smoke concentrations across Europe: results from the PEACE study. Atmos Environ 1997; 31: 3609-3622.

10. Hoek G, Roemer W, Brunekreef B. Methods for measurement of concentrations of PM10, black smoke, $\mathrm{SO}_{2}$ and $\mathrm{NO}_{2}$ in outdoor air in the framework of the PEACE study. Report, Dept of Epidemiology and Public Health, Wageningen Agricultural University, 1996.

11. DerSimonian R, Laird N. Meta-analysis in clinical trials. Controlled Clin Trials 1986; 7: 177-188.

12. Houwelingen JC. Meta-analysis. Cursus Boerhaave. Commissie voor post academisch onderwijs in de geneeskunde. Leiden, November 121993 (in Dutch).

13. Berlin JA, Longnecker MP, Greenland S. Meta-analysis of epidemiologic dose-response data. Epidemiology 1993; 4: $218-228$.

14. Timonen KL, Schwartz J, Nielsen J, et al. Chronic respiratory symptoms, skin prick tests results and lung function as predictors of acute respiratory symptoms. Am J Respir Crit Care Med 1998; 157: A642.

15. Timonen KL, Pekkanen J. Air pollution and respiratory health among children with asthmatic or cough symptoms. Am J Respir Crit Care Med 1997; 156: 546-552.

16. Vedal S, Schenker MB, Munoz A, Samet JM, Batterman S, Speizer FE. Daily air pollution effects on children's respiratory symptoms and peak expiratory flow. Am J Public Health 1987; 77: 694-698.

17. Hoek G, Brunekreef B. Effects of low level winter air pollution concentrations on respiratory health of Dutch children. Environ Res 1994; 64: 136-150.

18. Dockery DW, Pope CA III. Acute respiratory effects of particulate air pollution. Annu Rev Public Health 1994; 15: $107-132$

19. Schwartz J, Wypij D, Dockery D, et al. Daily diaries of respiratory symptoms and air pollution: methodological issues and results. Environ Health Perspect 1991; 90: 181-187.

20. Hastie TJ, Tibishirani RJ. Generalized Additive Models. London, Chapman and Hall, 1990.

21. Collier AM, Pimmel RL, Hasselblad V, Clyde WA, Knelson JH, Brooks JG. Spirometric changes in normal children with upper respiratory infections. Am Rev Respir Dis 1978; 117: 47-53.

22. Katsouyanni K, Touloumi G, Spix C, et al. Short term effects of ambient sulphur dioxide and particulate matter on mortality in 12 European cities: results from time series data from the APHEA project. BMJ 1997; 314: $1658-1662$.

23. OECD. Methods of measuring air pollution. Report of the working party on methods of measuring air pollution and survey techniques. Paris, OECD, 1964.

24. Chow JC. Measurement methods to determine compliance with ambient air quality standards for suspended particles. J Air Waste Manage Assoc 1995; 45: 320-382.

25. Horvath $\mathrm{H}$. Discussion. Black smoke as a surrogate for PM10 in health studies. Atmos Environ 1996; 30: 26492650. 